\title{
Theoretical atomic radii of elements (H-Cm): A non-relativistic study with Gaussian basis set using HF, post-HF and DFT methods
}

\author{
Krishnamohan G. Prasanna ${ }^{\ddagger *}$, Sooraj Sunil ${ }^{\S}$, Ajith Kumar ${ }^{\S}$, James. T. Joseph ${ }^{\S}$ \\ $\ddagger$ Department of Science and Humanities, Mar Baselios College of Engineering and Technology, \\ Trivandrum, Kerala 695015, India. \\ $\S$ Department of Chemistry, Mar Ivanios College (Autonomous), Trivandrum, Kerala 695015, \\ India.
}

E-mail: krishna.mohan@mbcet.ac.in 


\begin{abstract}
We calculated the most probable radius of an atom for elements from $\mathrm{H}$ to $\mathrm{Cm}$. The calculations were carried out by using non-relativistic, spin polarized, HF, MP2 and DFT methods with all electron Gaussian basis set. Periodicity of atomic radii was correlated with the experimental first ionization energies. This non-relativistic atomic radii were also compared with other theoretical atomic radii. With respect to the Dirac-Slater data, our values were in good agreement with the elements up to Sn. Relationship with van der Waals radii of noble gases was discussed. Anomalous properties of $\mathrm{Gd}$ and Pd were examined. Linearity of lanthanide contraction of elements with $4 f$ electrons is illustrated. This linearity is found independent of the extent of electron correlation.
\end{abstract}

\title{
Introduction
}

Atomic radius - principally, a measure of the atomic size - is not a clearly defined quantity. However in practice, the atomic radius can be classified using ionic, covalent, metallic, and van der Waal's radii and they can be related to many observable quantities such as ionization energy, electron affinity, and strength of the chemical bond [1-3]. Lanthanide or actinide contraction is a known fact that directly related to the atomic size [4]. The Fajans' rule, which is related to the size of atoms, is used to determine whether a chemical bond is expected to be predominantly ionic or covalent. In computational materials science, the quality of pseudopotential can be evaluated by comparing its cut-off radius with the most probable radius of a valence electron(s) wavefunction [5].

Compared to experimental atomic radii, the theoretical atomic radii - the most probable radii or the radius corresponds to the outermost peak in the radial distribution of electrons - is well defined concept in quantum chemistry (here the symbol, $R_{\max }$, is used to represent it). And numerically, it can be calculated by solving the electronic Schrodinger equation of single, neutral atom by using Hartree-Fock (HF), post Hartree-Fock, or density functional theory (DFT) methods. Figure 1 presents an example, where the radial distribution functions or RDFs of $1 s, 2 s$, $3 s, 3 p, 3 d$, and $4 s$ electrons of the $S c$ atom is shown (calculated with PBE1PBE functional and UGBS basis set; see the computational methods section for more details). Here $R_{\max }$ is defined as the radius corresponding to maximum of the radial distribution function of $4 \mathrm{~s}$ electron. 
The first $R_{\max }$ data of neutral atoms was tabulated by Slater based on non-relativistic HF calculations [6]. However, Clementi and Raimondi [7, 8] conducted the most cited study (ca. 2000 citations) on theoretical atomic radii. They used the HF method with a minimum STO type basis set without any relativistic correction to obtain various data including $R_{\max }$; however, use of nodeless Slater functions lead only approximate solutions of radial wavefunctions. Waber and Cromer performed relativistic Dirac-Slater calculations and obtained $R_{\max }$ values similar to those of Clementi [9]. The importance of the relativistic effects (such as the contraction of $s$ and $p$ electrons) was reported, and this calculation was based on the local density approximation. Desclaux [10] used the Dirac-Fock equation to incorporate relativistic corrections for heavier elements. This study showed the importance of relativistic corrections for core and valence inner electrons for medium-sized to heavy atoms. Furthermore, this study also employed a nonrelativistic HF calculations to compute $R_{\max }$. Note that, all the aforementioned methods used integro-differential equations, to calculate total atomic [11] energies and radial wavefunctions instead of the matrix version of Schrodinger equation. Additionally, all these methods do not account the Coulomb electron correlation.

Rahm et al. [1] reported atomic radii (from $H$ to $\mathrm{Cm}$ ) by using relativistic DFT with $P B E 1 P B E$ functional [12] and a highly accurate basis set (ANO-RCC, See ref. [13]). The metric adopted in their study was the average distance from the nucleus, where the electron density decreases to 0.001 electrons per bohr ${ }^{3}$. This metric was originally proposed by Boyd [1] who showed that the relative radii of different atoms are nearly invariant with a further decrease in the cutoff density of 0.001 electrons per bohr ${ }^{3}$. Since this radii are not directly related to wavefunctions, its values were considerably different from those of most probable radii; however, these values well correlated with the van der Waals radii derived from crystal structures [3]. Note that at this van der Waals radius, an atom begins to interact with the valence electrons of other atoms [14] .

Guerra et al. published the Dirac-Fock calculations [15] of the effective nuclear charge and radii for elements $(\mathrm{Z} \leq 118)$ and compared the values with those of Clementi. They have found that disparities were small for $3 d$ elements and increased for $4 d$ and $5 d$ elements. Gosh et al. [2] quantitatively related the radii of elements with the experimental ionization energy of elements and screening constants (which were obtained either from spectroscopic parameters or 
HF level theory). With an effective nuclear charge $\left(Z_{\text {eff }}\right)$ and ionization energy $(I)$ the most probable radius of a single and neutral atom can be expressed (with fitted constants, $a, b, c$ ) as follows:

$$
R_{\max }=\frac{\mathrm{a}}{\mathrm{I}}+\frac{\mathrm{b}}{\mathrm{Z}_{\mathrm{eff}}}+\mathrm{C}
$$

Because $|a|>2|b|$ in this equation, the atomic radius depends more on ionization energy than the effective nuclear charge does. Hence, the radius can be predominantly, but inversely, correlated to ionization energy.

Moreover, Szarek [16] introduced another quantitative expression of $R_{\max }-$ in which the most probable radius can be expressed as the square root of polarizability $(\alpha)$ and hardness $(\eta)$ as follows:

$$
R_{\max } \sim \sqrt{\alpha \eta}
$$

where, chemical hardness can be defined in terms of ionization energy (IE) and electron affinity, (EA), as:

$$
\eta=\frac{1}{2}(\mathrm{IE}-\mathrm{EA})=\frac{1}{2}\left(\mathrm{E}_{\mathrm{LUMO}}-\mathrm{E}_{\mathrm{HOMO}}\right) .
$$

In this context, $R_{\max }$ corresponds to the most probable distance between the HOMO electron and nucleus. This radius exhibits an excellent correlation with the results of Cordero [17].

In this study, we calculated the most probable radii of atoms up to 96 elements $(\mathrm{H}-\mathrm{Cm})$ of the periodic table. We derived the radii by using non-relativistic and spin unrestricted HF, post-HF (MP2), and DFT calculations. To the best of our knowledge, this study is the first theoretical atomic radii calculation, which uses (all-electron-) Gaussian basis [18]. 


\section{Computational Details}

We used Gaussian electronic structure program (G16) for all of our calculations [19]. For nonrelativistic calculation we used spin-unrestricted HF, MP2 and DFT methods in conjunction with the UGBS basis set. It should be noted that at present only few all-electron basis set are available to span a sizable range of elements.

It is well known that the MP2 technique is a size-consistent method and gives the correlation energy correction to the second order [20]. Although the DFT and MP2 are conceptually different methods, usually ground state level DFT calculation gives similar accuracy of MP2 [21]. The functional used in our DFT calculation is PBE1PBE - a generalized gradient approximated hybrid functional of PBE functional and in contrast to other well-known GGA functional (e.g.PW91) this functional is constructed using only fundamental constants and the few parameters of local density approximation

Note that all the previous studies to obtain $R_{\max }$ values, non-Gaussian basis sets were used. And most of our atomic SCF calculations were converged to the experimental ground state configuration; but for few elements (irrespective of methods) the calculations didn't produce the exact ground state configuration. In order to rectify this issue we either used the initial wavefunction which exactly matches the experimental electron configuration (by transposing the appropriate orbitals) or by applying non-default initial guesses (such as Huckel, INDO) as starting wavefunction.

We obtained $R_{\max }$ from RDF (i.e. from the converged total wavefunctions, which include spherical harmonics). And by using normalized spherical harmonics function, one can write,

$$
\mathrm{RDF}=\left[\mathrm{R}_{\mathrm{nl}}(\mathrm{r})\right]^{2} \mathrm{r}^{2} \int_{\theta=0}^{\pi} \int_{\phi=0}^{2 \pi} \mathrm{Y}_{\mathrm{lm}}(\theta, \varnothing) \mathrm{Y}_{\mathrm{lm}}(\theta, \varnothing) \sin (\theta) \mathrm{d} \theta \mathrm{d} \varnothing=\left[\mathrm{R}_{\mathrm{nl}}(\mathrm{r})\right]^{2} \mathrm{r}^{2}(2)
$$

Note that $R D F(r) d r$ represents the probability of finding an electron in the volume between two concentric spheres of radius $r$ and $r+d r$. 
From the definition of RDF, one can write that:

$\int_{0}^{\infty} \mathrm{RDF}_{\mathrm{nl}} \mathrm{dr}=1$

In other words, $R_{n l}(\boldsymbol{r})$ is calculated from the converged atomic orbital coefficients followed by a spherical averaging (a numerical integration over $\theta$ and $\varnothing_{\text {variables; See equation 2) }}$ to obtain angular independent radial wavefunction. After obtaining the radial wavefunction (for different $n$ and $l$ ) RDF can be calculated. Note that the last equation can be used to test the quality of the numerical integration scheme. Because of numerical integration, RDF usually contains some amount of numerical noise. This noise data is found to be diminished if one uses smaller values of integration steps for $\theta$ and $\varnothing$ variables. We used a fitting procedure, polynomial regression, to eliminate the effect of noises in the RDF data.

Compared to atomic codes [11], the SCF converged wavefunction data from Gaussian does not explicitly tells the ground state configuration of the atom (in terms of principal, angular and magnetic quantum numbers). So that, in our calculations, the ground state configuration of atoms were matched with that of experimental state by applying, (1) aufbau principle, and (2), by analyzing radial nodes and symmetry of spherical harmonics part of the total wavefunction.

We restrict our study with non-relativistic DFT methods since our relativistic DFT study based on ANO-RCC basis set using DKH2 Hamiltonian (with G16 program suite) were not converged into the experimental ground state configuration for elements from $\mathrm{Y}-\mathrm{Cm}$.

\section{Results and Discussions}

As expected, we have found general characteristics of the periodicity in the $R_{\max }$ values (eg. alkali metal elements show largest radii along a period, and conversely, the noble gas elements show smallest radii across a period. Similarly, the size of atom is decreased as one moves along a period). In all our non-relativistic calculations the smallest and largest atom are found to be helium and francium, respectively (See Table S1). This can be readily correlated with its experimental first ionization energies (IE). However the density based metric [1] found the smallest and largest atoms as $\mathrm{Ba}$ and Ac, respectively. By comparing $R_{\max }$ with experimental IE, it is found that on an average, $R_{\max }$ (obtained from HF and MP2) is inversely proportional (IE ${ }^{-1.23}$ ) 
to the first ionization of energy (See Fig.S1-3). This qualitatively justifies Ghosh's ansatz (eqn.1).

We have noted a significant similarity between the radii obtained from HF and MP2 methods (for $s$ and $p$ block elements). See Fig.2 and FigS4. This similarity of is due to the fact that, the radial probability density or, simply, the electron density of the HF method is correct to second order $[22,23]$ and thus one can consider the HF theory as a good approximation to the exact density [24].

And it is clear from the Fig.S4 that $R_{\max }$ values from PBE and MP2 (or HF) match well with $s$ and $p$ block elements but not with $d$ and $f$ block atoms (as it shows over binding of electrons and hence the smaller $R_{\max }$ values). It is known that, in general, DFT performs good enough for main group elements with respect to transition metal elements [25], and PBE1PBE functional give least accurate ionization potential $\left(B 3 L Y P \_R M S E=2 \mathrm{kcal}\right.$ mol PBE1PBE_RMSE $=5.5 \mathrm{kcal}$ mol1, RMSE is root mean square errors, See [26] for additional discussions).

The deviation of our result with that of Clementi is increased if one move from $d$-block to $4 f$ elements. But from elements, $Z<20$, the deviation is negligibly small. This account a fact that the Clementi's double-zeta basis set is good enough to describe smaller systems but for $3 d$ and higher elements, one has to use more accurate basis set. However, the Clementi's basis set is, in essence, a Slater type basis set (unlike a Gaussian basis) so only less number of functions is needed to get good converged values.

Descalux's data was compared with our data. Note that Descalux also used a Slater type basis function. Here, Dirac-Slater equations were used for relativistic calculations whereas HF equations were applied for non-relativistic calculations and both of these methods does not treat the Coulomb correlation. The non-relativistic result (i.e. HF data of $R_{\max }$ ) of ours and that of Desclaux is matched very well for $Z<90$ (See Fig.S5 and Fig.2). But for relativistic calculation [15], the numbers were matched from $Z<50$, approximately. It should be stressed that the value of Descalux and Guerra is very similar - since they used similar method (Dirac-Fock) and basis set (Slater). 
Although there is no experimental method exists, as far as we know, to measure the size of a single or isolated neutral gaseous atom, the electron diffraction experiments [27] can be used to estimate a most-probable location of valence shell electrons especially for lighter elements. From hydrogen to argon, it gives a good correlation with the total HF electron density. But beyond argon, the theoretical as well as experimental electron density distribution doesn't give rise to new local maxima (which corresponds to the $\mathrm{N}$ shell) or a corresponding $R_{\max }$ value [28, 29]. This is mainly attributed to the wider broadening of the radial wave function of the outer electrons in the valence shells.

For noble gas atoms, the experimental van der Waals radii (See [30, 3, 17] ) values are found to be correlated with the theoretical values and a good linear correlation was obtained. Note that the values reported by Alvarez are always higher than that of Zhang's, and it is because of the different coordination number (12 and 6 for Alvarez's and Zhang's data set, respectively, and as expected, atomic radii increases with the coordination number). Interestingly the Zhang's interpolated values were found to be well correlated with our MP2 values (in this case the coefficient of determination or, $R^{2}$, is 0.98 , see Fig. 3). It is primarily because of an assumption in the measurement - the radii of a noble gas atom greatly depend only on the interaction of its nucleus and electrons and not on the interaction between the nucleus and its nearest neighbor nucleus. Since the $R_{\max }$ is well matched with this experimentally fitted curve one can validate that the radius of noble gas element is primarily depending on the electrostatic interaction of nucleus and its outermost electrons. Experimentally deduced radii of Alvarez also show good correlation with our MP2 values $\left(R^{2}=0.81\right)$.

We compared our values with the radii of Rahm et. al. which is based on relativistic-DFT (the metric adopted here is related to the density of a free and neutral atom and not directly related to the radial wavefunction). A fairly good correlation was found $\left(R^{2}=0.75\right.$, with respect to our $\mathrm{PBE}$ data) and in all cases our values are found to be smaller. Interestingly, by omitting alkali atoms the $R^{2}$ value was raised up to 0.88 . From Fig. 1 it is clear that $s$ radial functions are generally very broad if $n>3$. Furthermore, this broadening is decreased if we move along a period. In Rahm's measure a density constraint is used (0.001 electrons per bohr ${ }^{3}$ ) and to satisfy this constraint, especially for these ns electrons, a larger radius should be considered. This will lead a larger discrepancy for alkali metals. From the comparison of experimental values (of Alvarez) it 
is found that Rahm's value is very closely related to the van der Waal's radii and it is not directly related to the single-atom radii generated from radial wavefunctions.

A striking deviation is observed for Pd in which $R_{\max }$ has a value of 0.586 Angstrom. In our calculation we have found that, it is the $4 d$ electron which gives $R_{\max }$ i.e. $4 \mathrm{~d}$ electron is the outermost valence electron - as it is seen from the experimental electronic state: $[\mathrm{Kr}] 4 \mathrm{~d}^{10}$. This deviation of radii is also reflected in the experimental ionization potential (See Fig.S6).

A gadolinium-break, that is, a discontinuity around the $\mathrm{Gd}$ atom as it is observed in several theoretical and experimental properties (such as ionic radii, constant of formation of Gdcomplexes [31], static dipole polarizability [32]) has also been clearly seen in our data. This 'break' in the atomic radius is also observed in the relativistic calculations [33]. This contraction of radius can be attributed to the weaker screening of $5 d$ electrons [14].

Seitz et.al, [34] investigated the lanthanoides-O bonds in a set of complex molecules and found that the bond lengths are decreased in a quadratic manner. They represented this quadratic decay with an equation,

$$
19.872-0.146 n+0.0034 n^{2}
$$

where, $n$ is the total number of $f$ electrons.

This decrement was modeled by Slater's model for ionic radii. Nevertheless, it should be noted that the small value of the quadratic component (0.0034) with respect to the linear component leaving a chance for linearity for the lanthanide contraction. A similar study by Quadrelli [35] also indicated a parabolic behavior of the contraction (in lanthanoids-O distance) in a set of lanthanide complexes. This apparent quadratic dependence is, however, interpreted differently [36].

In our study, a linear decrement of radii (of $6 \mathrm{~s}$ electrons) has been observed for lanthanides having $[\mathrm{Xe}] 4 f^{n} 6 s^{2}$ configuration - irrespective of the computational methods (which accounts various levels of electron correlation). And this linearity can be expressed in terms of $n$ as:

$$
R_{H F / M P 2}=-0.025 n+2.34
$$




$$
R_{P B E}=-0.025 n+2.28
$$

A similar linear trend is also visible in the relativistic calculations [10, 15, 33] and interestingly in the experimental first ionization energies (Fig.S6). The correlated data of $\mathrm{Ln}^{+3}$ ionic radii (i.e. metal ions with a $[\mathrm{Xe}] 4 f^{n}$ configuration) and $R_{\max }$ also shows a comparable linearity (Fig.S7). In other words, the presence of $5 d$ electrons considerably alters this linearity.

\section{Conclusion}

In this study, we presented the non-relativistic, most probable radius of the outermost electron of an atom (H-Cm) by using the radial wavefunctions obtained from all-electron Gaussian basis set. We used spin unrestricted non-relativistic Hartree-Fock, post Hartree-Fock (MP2), and density functional theory methods to calculate the radial wavefunctions. Experimental van der Waals radii of inert gases were related with our data, and found a good agreement between these values. Correlation studies, with other theoretical data and experimental first ionization energy, were carried out. Linearity in atomic lanthanide contraction with a discontinuity at gadolinium was observed. This linearity is found independent of the extent of electron correlation.

\section{Acknowledgements}

KGP thanks Google’s academic research credits program for providing computing resources.

\section{References}

1. Rahm M, Hoffmann R, Ashcroft NW (2016) Atomic and lonic Radii of Elements 1-96. Chemistry 22: 14625-14632. https://doi.org/10.1002/chem.201602949

2. Chakraborty T, Gazi K, Ghosh DC (2010) Computation of the atomic radii through the conjoint action of the effective nuclear charge and the ionization energy. Molecular Physics 108: 20812092. https://doi.org/10.1080/00268976.2010.505208

3. Alvarez S (2013) A cartography of the van der Waals territories. Dalton Trans 42: 8617-8636. https://doi.org/10.1039/C3DT50599E

4. Cotton F. A., Wilkinson G., Murillo C. A., Bochmann M. (2009) Advanced Inorganic Chemistry. Wiley-NewYork

5. Payne MC, Teter MP, Allan DC et al. (1992) Iterative minimization techniques for ab initio totalenergy calculations: molecular dynamics and conjugate gradients. Rev Mod Phys 64: 1045-1097. https://doi.org/10.1103/RevModPhys.64.1045 
6. Slater JC (1930) Atomic Shielding Constants. Phys Rev 36: 57-64. https://doi.org/10.1103/PhysRev.36.57

7. Clementi E, Raimondi DL (1963) Atomic Screening Constants from SCF Functions. The Journal of Chemical Physics 38: 2686-2689. https://doi.org/10.1063/1.1733573

8. Clementi E, Raimondi DL, Reinhardt WP (1967) Atomic Screening Constants from SCF Functions. II. Atoms with 37 to 86 Electrons. The Journal of Chemical Physics 47: 1300-1307. https://doi.org/10.1063/1.1712084

9. Waber JT, Cromer DT (1965) Orbital Radii of Atoms and Ions. The Journal of Chemical Physics 42: 4116-4123. https://doi.org/10.1063/1.1695904

10. Desclaux JP (1973) Relativistic Dirac-Fock expectation values for atoms with $Z=1$ to $Z=120$. Atomic Data and Nuclear Data Tables 12: 311-406. https://doi.org/10.1016/0092-640X(73)90020-X

11. Froese-Fischer C, Brage T, Jönsson P (1997) Computational atomic structure: an MCHF approach. Institute of Physics Publishing, Bristol and Philadelphia

12. Adamo C, Barone V (1999) Toward reliable density functional methods without adjustable parameters: The PBEO model. The Journal of Chemical Physics 110: 6158-6170. https://doi.org/10.1063/1.478522

13. Roos BO, Lindh R, Malmqvist P-Å et al. (2005) New relativistic ANO basis sets for actinide atoms. Chemical Physics Letters 409: 295-299. https://doi.org/10.1016/j.cplett.2005.05.011

14. Tatewaki H, Hatano Y, Noro T et al. (2015) Atomic radii for atoms with the $6 \mathrm{~s}$ shell outermost: The effective atomic radius and the van der Waals radius from $55 \mathrm{Cs}$ to $80 \mathrm{Hg}$. AlP Advances 5: 67142. https://doi.org/10.1063/1.4922953

15. Guerra M, Amaro P, Santos JP et al. (2017) Relativistic calculations of screening parameters and atomic radii of neutral atoms. Atomic Data and Nuclear Data Tables 117-118: 439-457. https://doi.org/10.1016/j.adt.2017.01.001

16. Szarek P, Grochala W (2014) Most probable distance between the nucleus and HOMO electron: the latent meaning of atomic radius from the product of chemical hardness and polarizability. J Phys Chem A 118: 10281-10287. https://doi.org/10.1021/jp507423p

17. Cordero B, Gómez V, Platero-Prats AE et al. (2008) Covalent radii revisited. Dalton Trans: 28322838. https://doi.org/10.1039/b801115j

18. Castro EVR de, Jorge FE (1998) Accurate universal Gaussian basis set for all atoms of the Periodic Table. The Journal of Chemical Physics 108: 5225-5229. https://doi.org/10.1063/1.475959

19. Frisch MJ, Trucks GW, Schlegel HB et al. (2016) Gaussian 16, Gaussian, Inc.: Wallingford, CT, 2009

20. Krishnan R, Pople JA (1978) Approximate fourth-order perturbation theory of the electron correlation energy. Int J Quantum Chem 14: 91-100. https://doi.org/10.1002/qua.560140109

21. Chong DP (ed) (1995) Recent advances in density functional methods. World Scientific, Singapore

22. Møller C, Plesset MS (1934) Note on an Approximation Treatment for Many-Electron Systems. Phys Rev 46: 618-622. https://doi.org/10.1103/PhysRev.46.618

23. Kern CW, Karplus M (1964) Analysis of Charge Distributions: Hydrogen Fluoride. The Journal of Chemical Physics 40: 1374-1389. https://doi.org/10.1063/1.1725321

24. Bartell LS, Gavin RM (1964) Effects of Electron Correlation in X-Ray and Electron Diffraction. J Am Chem Soc 86: 3493-3498. https://doi.org/10.1021/ja01071a019 
25. Chan B, Gill PMW, Kimura M (2019) Assessment of DFT Methods for Transition Metals with the TMC151 Compilation of Data Sets and Comparison with Accuracies for Main-Group Chemistry. J Chem Theory Comput 15: 3610-3622. https://doi.org/10.1021/acs.jctc.9b00239

26. Cramer CJ, Truhlar DG (2009) Density functional theory for transition metals and transition metal chemistry. Phys Chem Chem Phys 11: 10757-10816. https://doi.org/10.1039/b907148b

27. Bartell LS, Brockway LO (1953) The Investigation of Electron Distribution in Atoms by Electron Diffraction. Phys Rev 90: 833-838. https://doi.org/10.1103/PhysRev.90.833

28. Sen KD, Slamet M, Sahni V (1993) Atomic shell structure in Hartree-Fock theory. Chemical Physics Letters 205: 313-316. https://doi.org/10.1016/0009-2614(93)89248-G

29. Boyd RJ (1976) The shell structure of atoms. J Phys B: At Mol Phys 9: L69-L72. https://doi.org/10.1088/0022-3700/9/5/001

30. Zhang Y, Z. Xu (1995) Atomic radii of noble gas elements in condensed phases. American Mineralogist 80: 670-675

31. Welch JM, Müller D, Knoll C et al. (2017) Picomolar Traces of Americium(III) Introduce Drastic Changes in the Structural Chemistry of Terbium(III): A Break in the "Gadolinium Break". Angew Chem Int Ed Engl 56: 13264-13269. https://doi.org/10.1002/anie.201703971

32. Thierfelder C, Schwerdtfeger $P$ (2009) Effect of relativity and electron correlation in static dipole polarizabilities of ytterbium and nobelium. Phys Rev A 79.

https://doi.org/10.1103/PhysRevA.79.032512

33. Pyykko P, Desclaux JP (1979) Relativity and the periodic system of elements. Acc Chem Res 12: 276-281. https://doi.org/10.1021/ar50140a002

34. Seitz M, Oliver AG, Raymond KN (2007) The lanthanide contraction revisited. J Am Chem Soc 129: 11153-11160. https://doi.org/10.1021/ja072750f

35. Quadrelli EA (2002) Lanthanide contraction over the $4 \mathrm{f}$ series follows a quadratic decay. Inorg Chem 41: 167-169. https://doi.org/10.1021/ic015580v

36. Raymond KN, Wellman DL, Sgarlata C et al. (2010) Curvature of the lanthanide contraction: An explanation. Comptes Rendus Chimie 13: 849-852. https://doi.org/10.1016/j.crci.2010.03.034 


\section{Figure Captions}

Fig.1 Radial distribution function (PBE) of Sc atom. Inset figure shows a $4 s$ RDF function and its $\mathrm{R}_{\max }$ located around $1.6 \AA$

Fig. 2 Different set of theoretical atomic radii $(\mathrm{H}-\mathrm{Cm})$

Fig.3 Correlation of experimental radii of inert gas with the $R_{\max }(M P 2)$ values

Fig.4 Linearity of $\mathrm{R}_{\max }$ of Lanthanide atoms with $[X e] 4 f^{n} 6 s^{2}$ configuration 